\title{
High-Efficiency Optical Gain in Type-II Semiconductor Nanocrystals of Alloyed Colloidal Quantum Wells
}

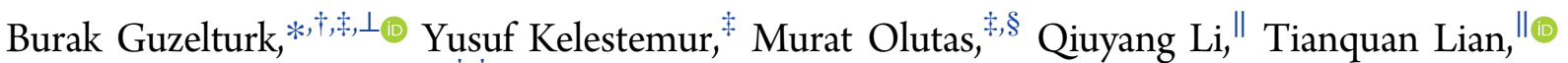 \\ and Hilmi Volkan Demir*, ${ }^{*}, \ddagger$ (†)
}

${ }^{\dagger}$ LUMINOUS! Center of Excellence for Semiconductor Lighting and Displays, School of Electrical and Electronic Engineering, School of Physical and Mathematical Sciences, Nanyang Technological University, Nanyang Avenue, Singapore 639798, Singapore

${ }^{\ddagger}$ Department of Electrical and Electronics Engineering, Department of Physics, UNAM - Institute of Materials Science and Nanotechnology, Bilkent University, Ankara 06800 Turkey

${ }^{\S}$ Department of Physics, Abant Izzet Baysal University, Bolu 14280, Turkey

"Department of Chemistry, Emory University, 1515 Dickey Drive, North East, Atlanta, Georgia 30322, United States

\section{Supporting Information}

ABSTRACT: Colloidal nanocrystals having controlled size, tailored shape, and tuned composition have been explored for optical gain and lasing. Among these, nanocrystals having Type-II electronic structure have been introduced toward low-threshold gain. However, to date, their performance has remained severely limited due to diminishing oscillator strength and modest absorption cross-section. Overcoming these problems, here we realize highly efficient optical gain in Type-II nanocrystals by using alloyed colloidal quantum wells. With composition-tuned core/alloyed-crown $\mathrm{CdSe} / \mathrm{CdSe} \mathrm{Te}_{1-x}$ quantum wells, we achieved amplified spontaneous emission thresholds as low as $26 \mu \mathrm{J} / \mathrm{cm}^{2}$, long optical gain lifetimes $\left(\tau_{\text {gain }} \approx 400 \mathrm{ps}\right)$, and high modal gain coefficients $\left(g_{\text {modal }} \approx 930 \mathrm{~cm}^{-1}\right)$. We uncover that the optical gain in these Type-II quantum wells arises from the excitations localized to the alloyed-

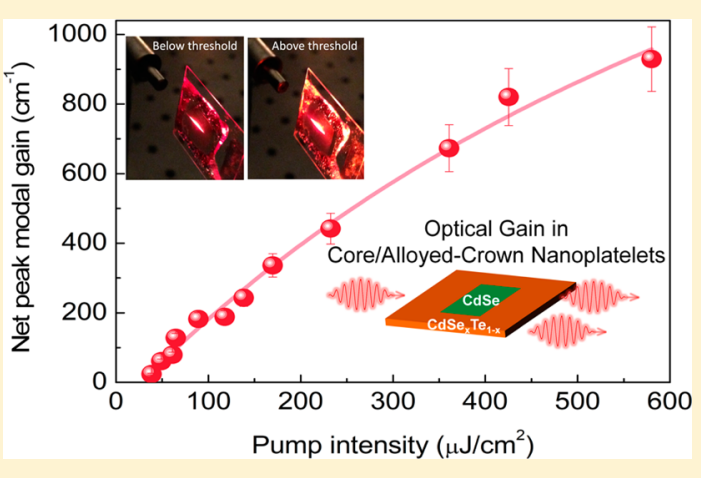
crown region that are electronically coupled to the charge-transfer state. These alloyed heteronanostructures exhibiting remarkable optical gain performance are expected to be highly appealing for future display and lighting technologies.

olloidal semiconductor nanocrystals hold great promise for full-color lasers ${ }^{1-7}$ as a potential game changer for future solid-state lighting and innovative display systems. date, numerous structures of nanocrystals with their shape tailored, composition tuned, and size controlled have been extensively investigated to aim for efficient optical gain performance. ${ }^{4,5,10-12}$ Yet their optical gain has been limited. This is a fundamental limitation arising due to the fact that single-exciton state does not provide net gain in common II-VI nanocrystals. To achieve net optical gain, biexcitons had to be generated by pumping these materials harder. ${ }^{3}$ However, biexcitons in conventional nanocrystals are very short-lived because they are rapidly annihilated via fast Auger recombination processes $(\sim 10-50 \mathrm{ps}),{ }^{13-16}$ immediately depleting the available optical gain and substantially shortening gain lifetime $\left(\tau_{\text {gain }} \ll 100 \mathrm{ps}\right)$.

Previously, heterostructured nanocrystals having Type-II electronic structure ${ }^{16,17}$ or soft-confinement potential ${ }^{1,2}$ have been proposed to circumvent Auger losses by either introducing single exciton gain or minimizing the Auger recombination process. However, optical gain performance has been commonly hampered in these nanocrystals due to reduced oscillator strength and small absorption cross-section, leading to overall modest stimulated emission cross sections and hence limited gain coefficients. Recently, colloidal quantum wells (CQWs), also known as colloidal nanoplatelets, ${ }^{18-20}$ have been shown to achieve superior optical gain performance among colloidal nanocrystals, resulting in low gain thresholds $^{21-24}$ and high gain coefficients. ${ }^{22}$ In these aforementioned works, only CQWs having either Type-I or quasi-Type-II electronic structure have been investigated. More recently, $\mathrm{CdSe} / \mathrm{CdTe}$ (core/crown) CQWs having pure Type-II electronic structure have been synthesized; these CQWs combine large Stokes-shifted emission with long fluorescence lifetimes, ${ }^{25-29}$ making them potentially interesting for amplified spontaneous emission (ASE) and lasing, which have remained elusive to date.

Here we demonstrate that Type-II CQWs that have composition-tuned alloyed-crowns exhibit remarkable optical gain performance with ASE threshold as low as $26 \mu \mathrm{J} / \mathrm{cm}^{2}$, accompanied by large net modal gain coefficients up to $\sim 930$ $\mathrm{cm}^{-1}$ and long gain lifetimes $\left(\tau_{\text {gain }} \approx 400 \mathrm{ps}\right)$. The performance

Received: September 6, 2017

Accepted: October 12, 2017

Published: October 12, 2017 
of the Type-II CQWs studied here represents more than an order of magnitude improvement over the previous best reports in Type-II colloidal nanocrystals. We corroborate the underpinning mechanism of this efficient optical gain by ultrafast transient absorption (TA) spectroscopy and show that the excitations localized to the alloyed-crown region, which are electronically coupled to the charge transfer (CT) excitations at the Type-II interface, are responsible for the gain. The electronic coupling between the crown excitations and the CT state results in strong energetic shift, allowing for the minimization of the reabsorption losses, which is otherwise detrimental for optical gain in conventional quantum dots, and enable four-level-like gain system.

CdSe core-only CQWs having a vertical thickness of $\sim 1.2$ $\mathrm{nm}$ (corresponding to four monolayers of CdSe with an additional Cd layer) and a lateral size of $14.0 \pm 1.6 \mathrm{~nm}$ by 12.7 $\pm 1.5 \mathrm{~nm}$ were synthesized. (See Figure S1 for the TEM image.) Using this core-only CQW as the seed, we synthesized core/alloyed-crown CQWs of CdSe/CdSe $\mathrm{Te}_{1-x}(x=0,0.25$, $0.50,0.75)$, where the crown layer grows laterally and the vertical thickness is kept fixed (see Methods). ${ }^{26,30}$ The lateral size of the core/alloyed-crown CQWs was analyzed using highangle annular dark-field scanning transmission electron microscopy (HAADF-STEM). Figure la,b shows the STEM images of the $\mathrm{CdSe} / \mathrm{CdSe}_{x} \mathrm{Te}_{1-x} \mathrm{CQWs}$ with $x=0$ and 0.50, respectively. (See Figure S2 for the other CQW samples.) After the crown growth, the lateral extent of the CQWs was uniform and the lateral size reached, on average, $29.3 \mathrm{~nm}$ by $20.6 \mathrm{~nm}$. (See Table S1 for the lateral sizes of the CQWs with different crown composition.)

To inspect the elemental composition of the alloyed-crown CQWs, we performed X-ray photoelectron (XPS) and energydispersive X-ray (EDS) spectroscopies. Table S2 compares the elemental compositions of the alloyed-crowns, estimated from the injected crown growth precursors and also analyzed via XPS measurements, showing a good agreement. Figure 1c depicts the line-probe EDS analysis of a single $\mathrm{CdSe} / \mathrm{CdSe} \mathrm{e}_{0.50} \mathrm{Te}_{0.50}$ CQW. (See Figure S3 for the CdSe/CdTe CQW.) The CdSe core and the $\mathrm{CdSe} \mathrm{e}_{0.50} \mathrm{Te}_{0.50}$ alloyed-crown regions can be clearly distinguished in the green and orange shaded areas, respectively. These observations strongly indicate the homogeneous lateral growth of the alloyed-crown with a well-estimated stoichiometry owing to the similar reactivity of the Se and Te precursors.

Figure 1d shows the UV-vis absorbance and steady-state photoluminescence (PL) spectra of the core-only and core/ alloyed-crown CQWs. Absorbance of the core-only CdSe CQW exhibits sharp excitonic features arising from the electron/heavy-hole (e/hh) and electron/light-hole (e/lh) transitions of CdSe at 512 and $480 \mathrm{~nm}$, respectively. ${ }^{21,31}$ This seed CQW is quite pure in terms of vertical thickness population, as we do not observe any emission or absorption feature belonging to another CQW population having a different vertical thickness. As alloyed-crown layers are laterally grown, new absorption features emerge. (See Figure 1d.) For example, the absorbance peak at $556 \mathrm{~nm}$ in the $\mathrm{CdSe} / \mathrm{CdTe}$ $\mathrm{CQW}$ is due to the e/hh transition in the CdTe crown. ${ }^{25}$ The absorbance features arising from the alloyed-crowns red shift as more Se is incorporated into the crown (see Figure 1d) due to the band gap bowing effect, which was previously observed in the alloyed $\mathrm{CdSe}_{x} \mathrm{Te}_{1-x}$ quantum dots. ${ }^{32}$

The steady-state PL emission of the CdSe/CdTe CQW peaks at $659 \mathrm{~nm}$ with a full width at half-maximum (fwhm) of
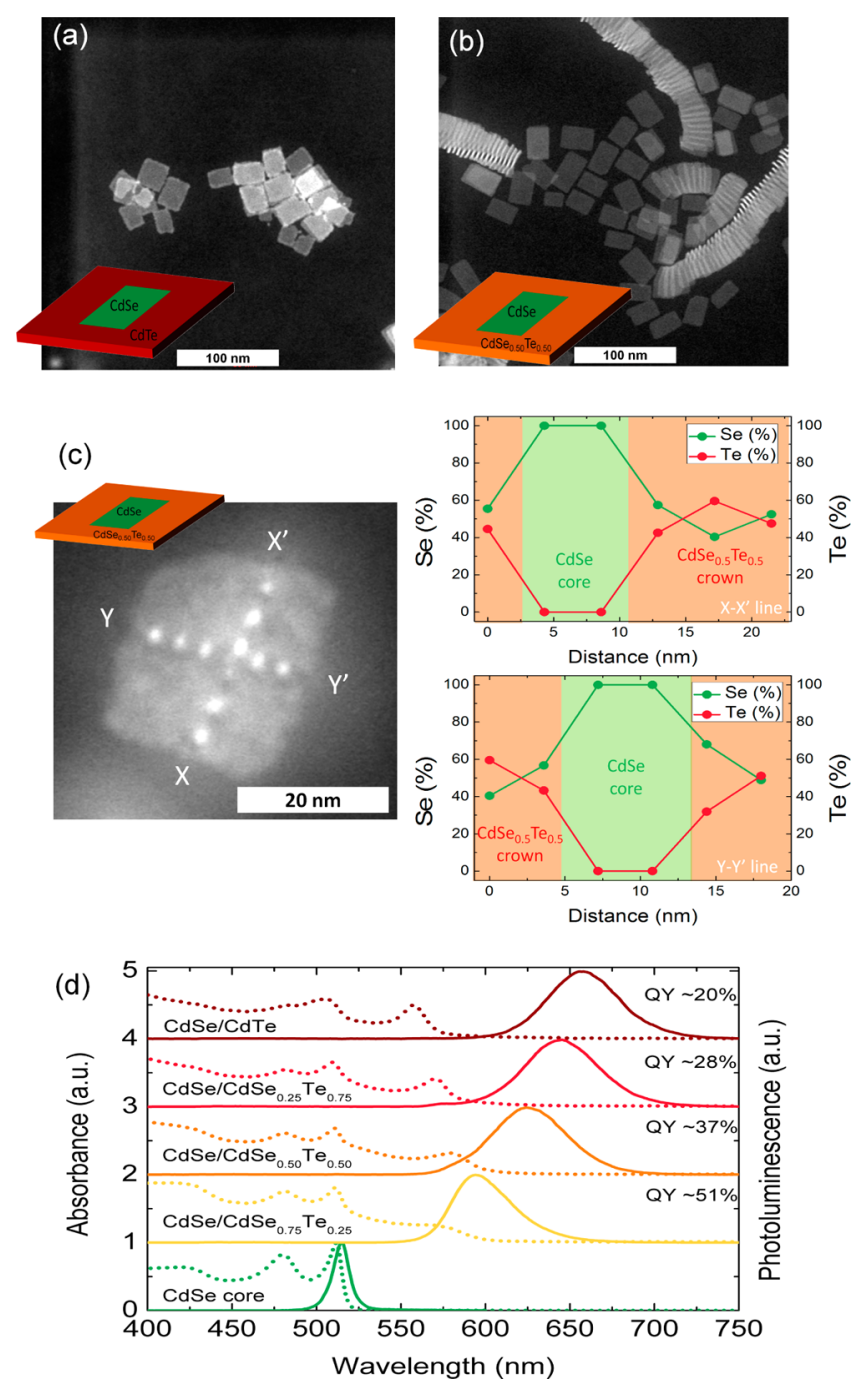

Figure 1. High-angle annular dark-field scanning transmission electron microscopy (HAADF-STEM) images of (a) CdSe/CdTe and (b) $\mathrm{CdSe} / \mathrm{CdSe}_{0.50} \mathrm{Te}_{0.50}$ CQWs. The scale bars are $100 \mathrm{~nm}$. (c) Energydispersive X-ray spectroscopy (EDS) on CdSe/CdSe $e_{0.50} \mathrm{Te}_{0.50} \mathrm{CQW}$ together with the analysis of the $\mathrm{Se}$ and $\mathrm{Te}$ fractions in the sample along the probed EDS lines of $\mathrm{X}-\mathrm{X}^{\prime}$ and $\mathrm{Y}-\mathrm{Y}^{\prime}$. The green and orange shaded areas show the regions of the core and the alloyed-crown, respectively. (d) Steady-state photoluminescence (solid) and absorbance (dotted) spectra of the core/crown Type-II CQWs along with those of the core-only CQW. Photoluminescence quantum yields (QYs) are also noted.

$49 \mathrm{~nm}$, arising from the spontaneous emission of the spatially indirect CT state across the Type-II interface. ${ }^{25,26,33}$ In the alloyed-crown CQWs, the PL emission peak blue-shifts as the fraction of $\mathrm{Se}$ in the crown is increased because the valence band edge energy is increased with more Se incorporated into the alloyed-crown. (See Figure S4 for the energy band alignments estimated from the absorbance and emission of the CQWs.) PL quantum yields are also noted in Figure 1d, which increase from 20 to $51 \%$ as the fraction of Se in the alloyed-crown is increased.

We investigated ASE in the thin films of the alloyed Type-II CQWs. For that, we tested both drop-casted and spin-coated films on quartz substrates. We found that spin-coated films (thickness $\sim 150 \pm 20 \mathrm{~nm}$ ) achieve lower ASE thresholds, possibly due to better film-packing and also smaller optical 

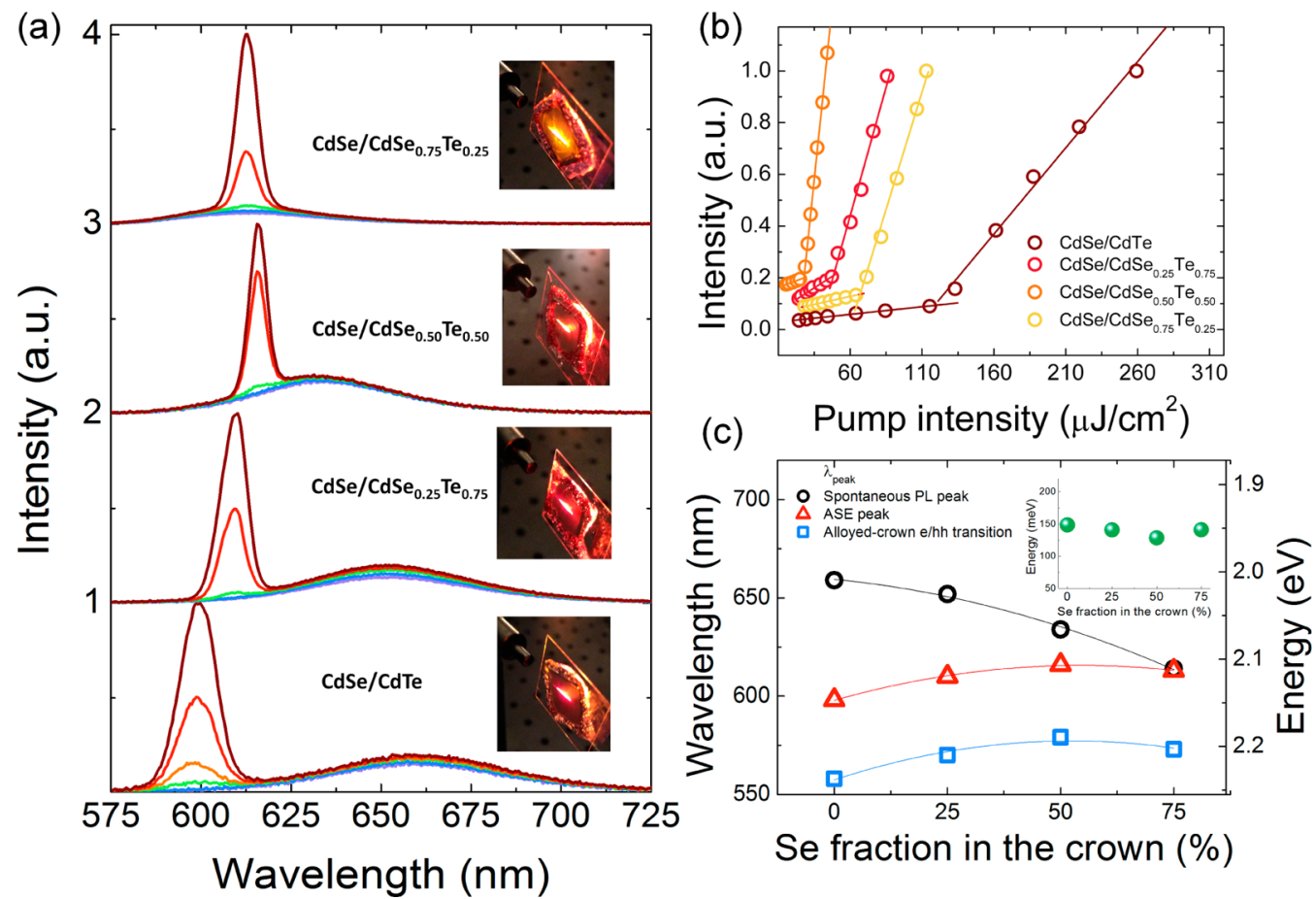

Figure 2. (a) Emission spectra of the Type-II CQWs as the pump intensity is increased, exhibiting the transition from spontaneous emission to amplified spontaneous emission (ASE). Insets show the photos of the thin films when the pump intensity is above the ASE threshold. (b) Pump intensity versus luminescence intensity in the Type-II CQW samples. The lowest ASE threshold $\left(26 \mu \mathrm{J} / \mathrm{cm}^{2}\right)$ is realized using the CdSe/ $\mathrm{CdSe}_{0.50} \mathrm{Te}_{0.50} \mathrm{CQWs}$. (c) Spectral position of the spontaneous emission peak (black circles), the ASE peak (red triangles), and the electron/heavyhole transition peak of the alloyed-crown (blue squares) as a function of the Se fraction in the crown. The inset shows the energy difference between the alloyed-crown's first exciton transition and the ASE peak. Constant energy difference suggests that the ASE peak spectrally follows the bandgap of the alloyed-crown.

waveguide losses. Therefore, we only studied the spin-coated films for the further investigations. To minimize sample degradation during ASE measurements, we encapsulated the samples in a nitrogen-filled glovebox using coverslips and applied epoxy to prevent contact with oxygen and moisture. (See Figure S5.) As the pump source, we employed frequencydoubled output $(400 \mathrm{~nm}$, using a BBO crystal) of a femtosecond Ti:sapphire regenerative amplifier (Spitfire Pro, Spectra Physics) having a $120 \mathrm{fs}$ pulse duration with a $1 \mathrm{kHz}$ repetition rate.

Figure 2a shows the PL spectrum of the CQW samples as the pump fluence is gradually increased, showing the transition from spontaneous emission into the ASE. The ASE peaks are observed to be blue-shifted with respect to their corresponding spontaneous emission peaks. The amount of blue shift changes with the alloyed-crown composition. For example, the ASE peak in the $\mathrm{CdSe} / \mathrm{CdSe}_{0.50} \mathrm{Te}_{0.50} \mathrm{CQW}$ emerges at $609 \mathrm{~nm}$, which is $\sim 19 \mathrm{~nm}$ blue-shifted with respect to the spontaneous PL emission peak. In Figure $2 b$, luminance intensity versus pump fluence is plotted. In the CQW having pristine CdTe crown, the ASE threshold is the largest $\left(119 \mu \mathrm{J} / \mathrm{cm}^{2}\right)$. We checked over 10 different $\mathrm{CdSe} / \mathrm{CdTe} \mathrm{CQW}$ samples having different core and crown sizes, where the ASE thresholds were in the range of $100-300 \mu \mathrm{J} / \mathrm{cm}^{2}$ with an average threshold of $\sim 200 \mu \mathrm{J} / \mathrm{cm}^{2}$ (see Figure S6), although the ASE peak was spectrally at the same position $( \pm 3 \mathrm{~nm})$. Therefore, it was not possible to further reduce the ASE thresholds in the pristine crown Type-II CQW. The alloyed-crown CQWs, on the contrary, exhibited remarkably improved ASE performance with much lower ASE thresholds. The alloyed-crown CQW in the form of $\mathrm{CdSe} / \mathrm{CdSe} \mathrm{e}_{0.50} \mathrm{Te}_{0.50}$ achieved the lowest ASE threshold $\left(I_{\mathrm{ASE}}=26 \mu \mathrm{J} / \mathrm{cm}^{2}\right)$. Also, we checked three different batches of $\mathrm{CdSe} / \mathrm{CdSe} \mathrm{e}_{0.50} \mathrm{Te}_{0.50} \mathrm{CQWs}$, which exhibited almost the ASE threshold $\left(25-30 \mu \mathrm{J} / \mathrm{cm}^{2}\right)$. The other alloyed-crown CQWs exhibited ASE thresholds of 48 and $66 \mu \mathrm{J} / \mathrm{cm}^{2}$ for Se fractions of 25 and $75 \%$, respectively. Therefore, alloyed-crown Type-II CQWs achieve substantially lower ASE thresholds than those of the nonalloyed-crown versions. Furthermore, slopeefficiency of the alloyed-crown CQWs is better than that of the pristine-crown CQW, suggesting effective utilization of the excitation for optical gain and hence higher gain coefficients.

Previously, the lowest ASE threshold in Type-II core/shell quantum dots was reported to be on the order of $2 \mathrm{~mJ} /$ $\mathrm{cm}^{2}{ }^{16,34}$ Therefore, the alloyed-crown Type-II CQWs here exhibit more than an order of magnitude improvement among Type-II nanocrystals. As a side note, ASE was not realized using Type-II CdSe/CdTe quantum dots before, possibly due to their strongly reduced oscillator strength in their core/shell quantum dot form. Therefore, Type-II nanostructures in the form of core/crown quantum wells are superior to their spherical core/ shell counterparts. Here the best ASE threshold is comparable to or better than the recently reported highly performing heterostructure-engineered nanocrystal systems including giantshell CdSe/CdS quantum dots $\left(I_{\mathrm{ASE}} \approx 29 \mu \mathrm{J} / \mathrm{cm}^{2}\right),{ }^{1,35} \mathrm{CdZnS} /$ $\mathrm{ZnS}$ quantum dots $\left(I_{\mathrm{ASE}} \approx 50 \mu \mathrm{J} / \mathrm{cm}^{2}\right),{ }^{4,5}$ and multishell CdSe/ $\mathrm{CdZnS} / \mathrm{ZnS}$ quantum dots $\left(I_{\mathrm{ASE}} \approx 90 \mu \mathrm{J} / \mathrm{cm}^{2}\right)$. $^{2}$ Also, our ASE threshold is on par with the average ASE threshold reported using Type-I (or quasi-Type-II) core/shell CdSe/CdS CQWs, ${ }^{21}$ where statistically averaged ASE threshold was $~ 24$ $\mu \mathrm{J} / \mathrm{cm}^{2}$, whereas their hero sample was reported to achieve 6 $\mu \mathrm{J} / \mathrm{cm}^{2}$. Similarly, giant-shell quantum dots have shown ASE thresholds down to $6 \mu \mathrm{J} / \mathrm{cm}^{2}{ }^{15}$ 
(a)

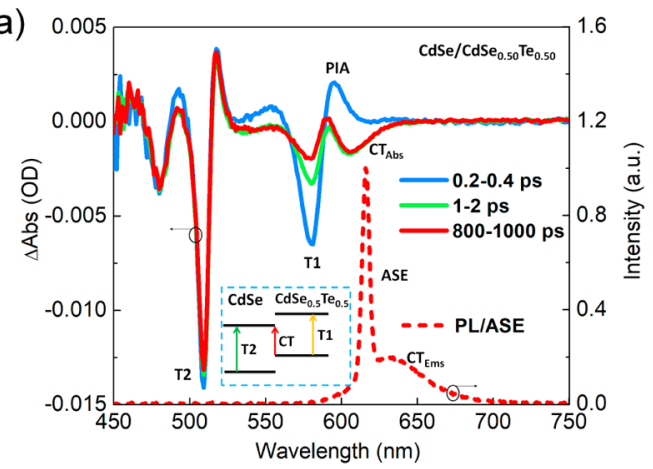

(c)

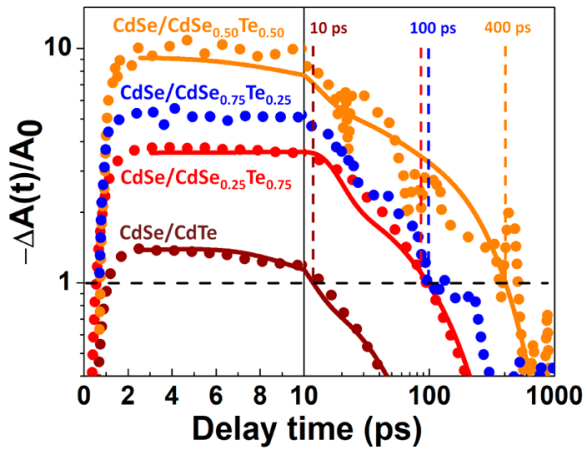

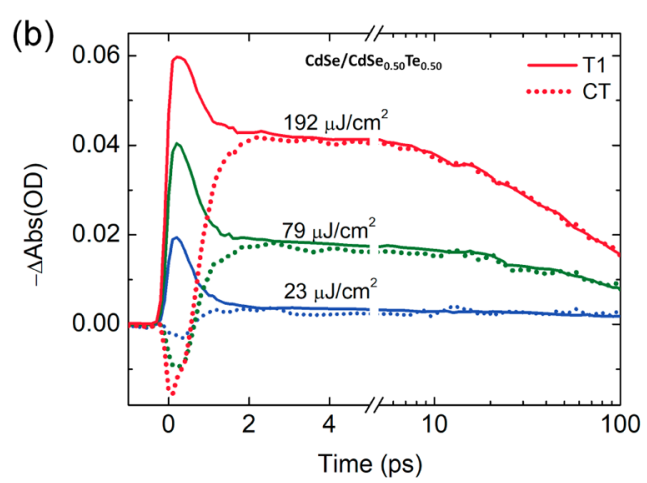

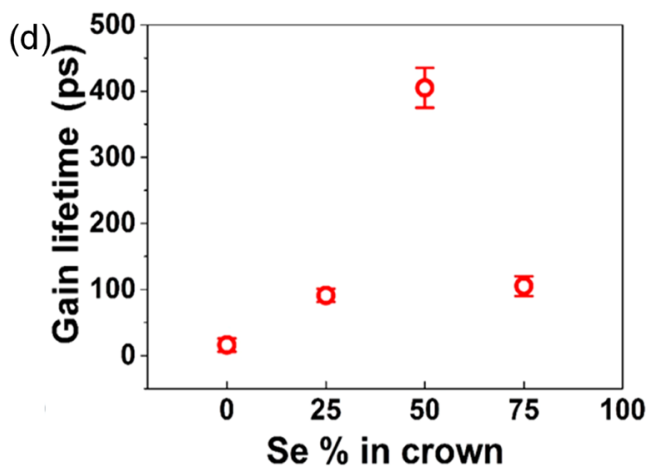

Figure 3. (a) Transient absorption (TA) spectra of CdSe/CdSe $e_{0.50} \mathrm{Te}_{0.50} \mathrm{CQW}$ at pump fluence of $79 \mu \mathrm{J} / \mathrm{cm}^{2}$ at pump-probe delays of $t=0.2-0.4$ ps, $t=1-2$ ps, and $t=800-1000$ ps. (b) Bleach recovery kinetics of the alloyed-crown's electron/heavy-hole transition (T1) (solid) and charge transfer $(\mathrm{CT})$ (dotted) in $\mathrm{CdSe} / \mathrm{CdSe}_{0.50} \mathrm{Te}_{0.50} \mathrm{CQWs}$ at selected pump fluences. (c) Normalized absorption $\left(-\Delta A(t=2.5 \mathrm{ps}) / A_{0}\right)$ as a function of the pump fluence measured for CdSe/CdSe ${ }_{x} \mathrm{CdTe}_{1-x}(x=0$ in brown, $0.25 \mathrm{in}$ red, 0.50 in orange, and 0.75 in blue $)\left(I_{\text {pump }} \approx 200 \mu \mathrm{J} / \mathrm{cm}^{2}\right)$. Above the dashed line indicates net optical gain. (d) Gain lifetimes in the alloyed Type-II CQWs as a function of the Se percentage in the crown.

Previously, in Type-II quantum dots (e.g., core/shell CdS/ $\mathrm{ZnS}$ ), optical gain has been shown to arise from both single excitons and also spatially indirect biexciton states. ${ }^{16,36}$ A blueshifted ASE was observed as attributed to the spatially indirect biexcitons, which possess positive binding energy $\left(E_{\mid X X}>-2 E_{\mid X>}\right.$ $=E_{\mathrm{B}}$ and $\left.E_{\mathrm{B}}>0\right)$ due to repulsive Coulomb interaction. In our case, if the ASE were to arise from spatially indirect biexcitons, then it would suggest the existence of a giant repulsive biexciton binding energy in these Type-II CQWs. For example, in the CdSe/CdTe CQW the blue shift between the ASE and the spontaneous emission peaks is $190 \mathrm{meV}$. (See Figure 2a.) Therefore, $\Delta E_{\mathrm{BX}}$ had to be on the order of $200 \mathrm{meV}$. However, Coulombic binding energy for an exciton in a CdSe CQW was theoretically calculated to be on the order of $150-200 \mathrm{meV}^{37}$ and was experimentally determined to be $180 \mathrm{meV}^{38}$ Considering the large lateral extent of the CQWs, which should reduce the exciton-exciton interactions, one would expect much lower $E_{\mathrm{BX}}$. Therefore, giant biexciton binding energy in Type-II CQWs does not seem likely to explain the origin of the gain, and hence the gain mechanism in the Type-II CQWs is different than what has been reported for the conventional Type-II quantum dots.

In Figure 2c, we show the spectral positions of the ASE peak (red triangles), spontaneous emission peak (black circles), and the e/hh transition of the crown (blue squares) in all CQW samples. Although energy difference between the ASE and the spontaneous emission peaks monotonically decreases, surprisingly, the ASE peak is found to spectrally follow the bandgap (e/hh transition) of the alloyed-crown. This observation indicates that optical gain is associated with the excitations that are localized to the alloyed-crown region.
To further investigate the presence of the excitations in the alloyed-crown, we performed ultrafast TA measurements. Previously, internal charge transfer within $\mathrm{CdSe} / \mathrm{CdTe} \mathrm{CQW}$ has been studied and CT state formation has been observed on a picosecond time scale. ${ }^{27}$ However, in this aforementioned study the excitation fluence was intentionally kept low to prevent the generation of more than one exciton per CQW. Therefore, the nature of the excitations and their spatial localization throughout the CQW under elevated pump conditions have not been understood. Here we carried out pump-probe TA spectroscopy in the alloyed-crown Type-II CQWs as a function of the pump fluence. Experimental details of the TA system are summarized in the Supporting Information. Figure $3 \mathrm{a}$ shows the TA spectra of the CdSe/ $\mathrm{CdSe}_{0.50} \mathrm{Te}_{0.50} \mathrm{CQW}$ (see Figure S7 for the other samples) (excited@400 nm with a fluence of $79 \mu \mathrm{J} / \mathrm{cm}^{2}$ ) at three different pump-probe delay times: just after the excitation $(t=$ $0.2-0.4 \mathrm{ps})$, at early time $(t=1-2 \mathrm{ps})$, and at later time $(t=$ $800-1000$ ps). Just after the excitation, we observe the bleach signals at 580 and $512 \mathrm{~nm}$ arising from the e/hh transitions of the $\mathrm{CdSe}_{0.50} \mathrm{Te}_{0.50}$ alloyed-crown (T1) and the CdSe core (T2), respectively, indicating the photogeneration of excitons in both the core and alloyed-crown regions. Also, a photoinduced absorption (PIA) feature is observed at $597 \mathrm{~nm}$ for early time, which marks the position of the biexciton state in the alloyedcrown. After a short time delay $(t=1-2 \mathrm{ps})$, the CT state is formed (appearing as bleach at $606 \mathrm{~nm}$ ) via electron (hole) transfer from the alloyed-crown (core) into the core (alloyedcrown). Interestingly, at long pump-probe time delay $(t=$ 800-1000 ps), both T1 and CT are found to coexist with persisting bleach signals at this fluence level $\left(79 \mu \mathrm{J} / \mathrm{cm}^{2}\right)$, which is expected to generate more than one exciton per CQW. T1 
(a)

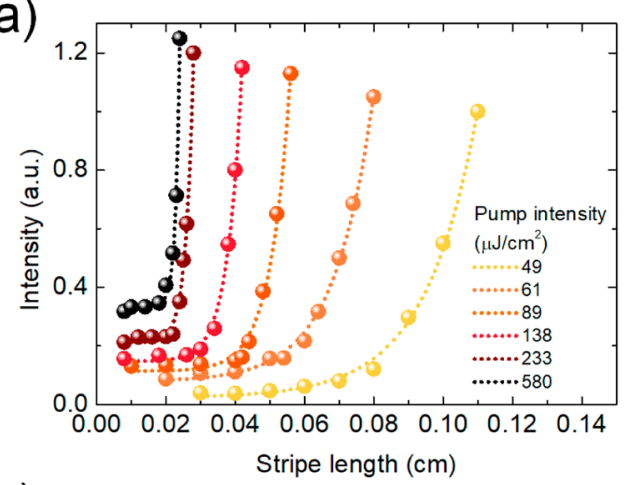

(c)

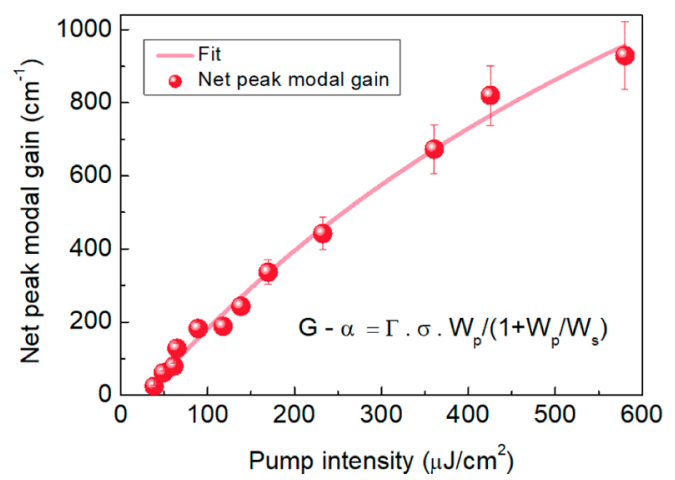

(b)

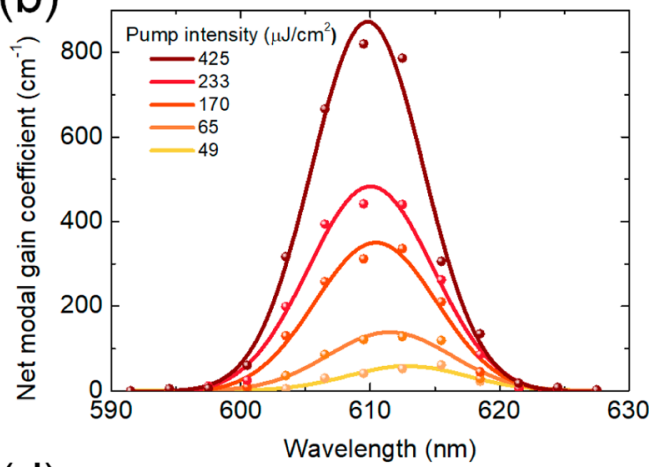

(d)

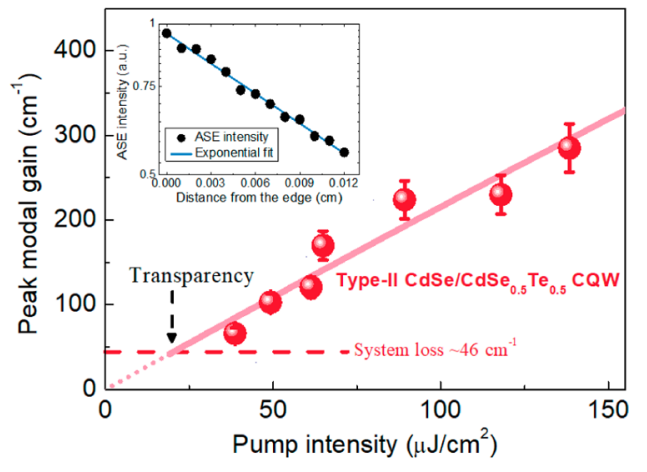

Figure 4. (a) Variable stripe length (VSL) measurement of the Type-II core/alloyed-crown CdSe/CdSe $e_{0.50} \mathrm{Te}_{0.50} \mathrm{CQW}$ under varying pump intensities fitted by eq 1. (b) Net modal gain spectra presented for increasing pump intensities $\left(50-420 \mu \mathrm{J} / \mathrm{cm}^{2}\right)$. The width of the gain spectrum is $\sim 9 \mathrm{~nm}$, the same as that of the ASE peak. (c) Net peak modal gain coefficients plotted and fitted by using eq 4 . The net gain $(G-\alpha)$ depends on the optical confinement factor $(\Gamma)$, stimulated emission cross-section $\left(\sigma_{\mathrm{st}}\right)$, pump intensity $\left(W_{\mathrm{p}}\right)$, and saturation pump intensity $\left(W_{\mathrm{s}}\right)$. The net modal gain coefficients increase linearly for pump intensities up to $200 \mu \mathrm{J} / \mathrm{cm}^{2}$, after which a gradual saturation occurs due to the saturating population inversion density. The maximum modal gain coefficient is $\sim 930 \mathrm{~cm}^{-1}$. (d) Peak modal gain coefficients $(G)$ plotted for the CdSe/CdSe $\mathrm{e}_{0.5} \mathrm{Te}_{0.5}$ CQW. System loss (waveguide loss factor) is found via measuring the ASE intensity as a function of the distance from the edge of the sample. (See the inset for the Type-II CQW sample.) The transparency point is achieved when the modal gain $(G)$ is equal to the waveguide loss coefficient $(\alpha)$.

transition persists for long time delays $(t>800 \mathrm{ps})$ even after the formation of the CT state. This indicates that excitations could persistently remain in the alloyed-crown even after initial CT across the Type-II interface.

In Figure $3 b$, we present the decay (or bleach recovery) kinetics of the T1 (solid) and CT (dotted) states under three different pump fluences. (See Figure S8 for the other CQW samples.) At a low pump fluence level (e.g., $23 \mu \mathrm{J} / \mathrm{cm}^{2}$ ), T1 has a fast decay component $(\sim 1 \mathrm{ps})$ that leads to the formation of the CT state, indicating an efficient CT state formation. On the contrary, T1 decay is observed to persist after a few picoseconds, suggesting that there seem to remain charges in the crown at long time delay. This becomes clearer at elevated pump fluences. At $79 \mu \mathrm{J} / \mathrm{cm}^{2}, \mathrm{~T} 1$ partially decays. While the fast decaying part forms the $\mathrm{CT}$ state, the remaining excitations in the crown decay slowly ( $\sim 100$ ps or longer). More importantly, as shown in Figure $3 b$, the decay kinetics of the remaining excitations in $\mathrm{T} 1$ and the $\mathrm{CT}$ state are found to be quite similar to each other. This indicates that both the CT state and the remaining excitation in the alloyed-crown are coupled via a shared electronic state (i.e., hole in the alloyedcrown). This coupling is expected to shift the energy levels of the excitations involved for the optical gain, and hence it can explain observed large spectral shift of the ASE peak with respect to the ground-state absorption (i.e., T1) and emission (i.e., CT state) in these Type-II CQWs.
In Figure $3 c$, we show the normalized absorption $(-\Delta A(t) /$ $\left.A_{0}\right)$ calculated at the ASE peak for each corresponding sample for a pump-probe delay of $t=2.5$ ps. The normalized absorption term being larger than 1 indicates the presence of net optical gain in the system. Optical gain lasts for only $\sim 10 \mathrm{ps}$ in the $\mathrm{CdSe} / \mathrm{CdTe} \mathrm{CQW}$, which explains the large ASE thresholds because gain active species are rapidly depleted before they could contribute to gain. The alloyed-crown CQWs, on the contrary, exhibit substantially longer optical gain lifetimes. Also, the gain amplitude is considerably larger in the alloyed-crown CQWs. We measured the net gain thresholds as $200,60,32$, and $65 \mu \mathrm{J} / \mathrm{cm}^{2}$ for the Se fraction of $0,0.25,0.50$, and 0.75 , respectively, by analyzing the fluence dependent normalized TA. (See Figure S9.) Therefore, the net gain thresholds excellently agree with the ASE thresholds. In Figure $3 \mathrm{~d}$, we depict the gain lifetimes for each sample. The best performing sample $\left(\mathrm{CdSe} / \mathrm{CdSe}_{0.50} \mathrm{Te}_{0.50} \mathrm{CQW}\right)$ attained a very long gain lifetime $\tau_{\text {gain }} \approx 400 \mathrm{ps,} \mathrm{almost} \mathrm{three} \mathrm{times} \mathrm{larger}$ as compared with the previous best reported measurements using heterostructured colloidal nanocrystals (i.e., core/shell $\mathrm{CQWs}^{39}$ and $\mathrm{CdSe} / \mathrm{CdS}$ nanorods ${ }^{40}$ ).

Next, we investigate the net modal gain coefficients in the alloyed-crown CQWs using variable stripe length (VSL) method as a function of the pump fluence. ${ }^{41}$ (See the Supporting Information for the experimental details.) Emission spectrum was measured as a function of the excitation stripe length, while the width of the stripe was kept fixed at $\sim 120 \mu \mathrm{m}$. 
Figure 4a shows the VSL data taken at the ASE peak at varying excitation intensities. To fit the VSL data, we employ the following relation that relies on $1 \mathrm{D}$ amplifier model ${ }^{42}$

$$
I(\lambda, l)=I_{\mathrm{S}} \frac{\left(\mathrm{e}^{(G(\lambda)-\alpha(\lambda)) l}-1\right)}{G(\lambda)-\alpha(\lambda)}
$$

Here $I(\lambda, l)$ is the measured intensity from the sample, $l$ is the stripe length, $I_{S}$ is the spontaneous emission proportionality constant, $G(\lambda)$ is the modal gain coefficient, and $\alpha(\lambda)$ is the waveguide loss coefficient. The term $(G(\lambda)-\alpha(\lambda))$ is the net modal gain coefficient. Equation 1 provides an excellent fit to the VSL data. (See Figure 4a.) In Figure $4 \mathrm{~b}$ we present the spectra of the net modal gain coefficients at increasing fluence levels, which could be fitted with a Gaussian function having a width of $\sim 9 \mathrm{~nm}$ (similar to the fwhm of the ASE peak). As the pump fluence is increased, the net modal gain coefficients grow and also slightly blue-shift, possibly due to the state-filling effect and multiexciton Coulomb interactions.

Figure $4 \mathrm{c}$ plots the net peak modal gain coefficients as a function of the pump fluence. At low pump fluence, net modal gain coefficient increases linearly, then a gradual saturation is observed at elevated excitation levels. To fit the net peak modal gain coefficients as a function of the pump fluence, we consider the following

$$
\begin{aligned}
& \text { net modal gain }=G-\alpha=\Gamma \times N_{\text {pop_inv }} \times \sigma_{\text {st }} \\
& N_{\text {pop_inv }}=\frac{W_{\mathrm{p}}}{1+\frac{W_{\mathrm{p}}}{W_{\mathrm{s}}}} \\
& G-\alpha=\Gamma \times \sigma_{\mathrm{st}} \times \frac{W_{\mathrm{p}}}{1+\frac{W_{\mathrm{p}}}{W_{\mathrm{s}}}}
\end{aligned}
$$

where $\Gamma$ is the optical mode confinement factor, $\sigma_{\text {st }}$ is the stimulated emission cross-section, and $N_{\text {pop inv }}$ is the density of the population inversion. The density of the population inversion can be expressed as in eq 3 , where $W_{\mathrm{p}}$ is the pump intensity and $W_{\mathrm{s}}$ is the saturation pump intensity. ${ }^{43}$ From the fit, we find $W_{\mathrm{s}}$ to be $1 \mathrm{~mJ} / \mathrm{cm}^{2}$ and $\alpha$ to be $\sim 50 \mathrm{~cm}^{-1}$. The net peak modal gain coefficient is as high as $929 \pm 23 \mathrm{~cm}^{-1}$ at $I_{\text {pump }}$ $=580 \mu \mathrm{J} / \mathrm{cm}^{2}$. Previously, the highest reported net modal gain coefficient among all types of colloidal nanocrystals was $\sim 900$ $\mathrm{cm}^{-1}$.22,44 Thus achievement of very large gain coefficients from the alloyed-crown Type-II CQWs makes them exciting not only for colloidal lasers but also for high-speed applications including optical switches.

To find the modal gain coefficients $(G)$, we measure the waveguide loss coefficient in these CQW thin films. For this, we measured the ASE intensity as a function of the distance from the edge of the sample. (See the inset of Figure $4 \mathrm{~d}$ and Supporting Information for the experimental details.) We fit the data by using the relation: $I_{\mathrm{ASE}}=I_{0} \mathrm{e}^{-\alpha l}$, where $I_{0}$ is the maximum ASE intensity and $l$ is the distance from the edge of the sample and $\alpha$ is the waveguide loss coefficient. Here $\alpha$ is found to be $46.0 \pm 1.2 \mathrm{~cm}^{-1}$, which is in good agreement with the fitting by eq 4 . Relatively high waveguide loss coefficient in the CQW film could be attributed to their needle-like face-toface assemblies, ${ }^{45}$ likely to increase the scattering losses. (See Figure S12.) We identify $\sim 22 \mu \mathrm{J} / \mathrm{cm}^{2}$ as the transparency excitation density. Furthermore, the extrapolation of the modal gain coefficients below the transparency point suggests that the modal gain coefficient starts increasing without a clear threshold (shown by the dotted line in Figure $4 \mathrm{~d}$ extrapolated to cross at the origin). This indicates that the Type-II CQWs could accomplish significantly lower gain thresholds if the waveguide loss coefficient (i.e., system loss) would be further reduced. Moreover, this gain behavior in the alloyed-crown CQWs, which resembles a four-level-like gain system, is expected to make them promising for ultralow threshold lasers based on low loss microcavities.

In conclusion, we explored optical gain in the CQWs having a Type-II electronic structure. Tuning the composition of the crown via alloying allowed us to realize exceptional optical gain performance in these colloidal nanocrystals. Also, we elaborated that the gain arises from the excitations in the crown that are coupled to the CT state at the Type-II interface. A deeper understanding of the crown's excitation-CT state coupling will be important for designing new colloidal heterostructures as ultraefficient optical gain media. These alloyed-crown quantumwell structures are expected to inspire novel colloidal gain media that will open up exciting revenues toward practical nanocrystal lasers.

\section{METHODS}

Synthesis of the $4 \mathrm{MLC}$ CdSe CQWs. CdSe nanoplatelets having four-monolayer $(4 \mathrm{ML})$ thickness were synthesized with the modified recipe from the literature. For a typical synthesis, 340 $\mathrm{mg}$ of cadmium myristate, $24 \mathrm{mg}$ of $\mathrm{Se}$, and $30 \mathrm{~mL}$ of octadecene (ODE) were loaded into a three-necked flask. The solution was degassed at room temperature for half an hour and heated to $240{ }^{\circ} \mathrm{C}$ under an inert atmosphere. When the temperature reached $195^{\circ} \mathrm{C}, 120-130 \mathrm{mg}$ of cadmium acetate dihydrate was injected into the reaction solution having bright yellowish color. After $10 \mathrm{~min}$ of the growth at $240{ }^{\circ} \mathrm{C}$, the temperature of the solution was decreased to room temperature with the injection of $1 \mathrm{~mL}$ of oleic acid (OA). With the purification of as-synthesized CdSe CQWs and the removal of other side-products including CQWs having different thicknesses and quantum dots, $4 \mathrm{ML}$ thick CdSe CQWs can be achieved having an emission peak around $513 \mathrm{~nm}$ with a moderately high quantum yield (30-50\%). Finally, 4 ML thick $\mathrm{CdSe}$ CQWs were dissolved in hexane and stored for next steps.

Preparation of Anisotropic Growth Mixture for $C d S e_{x} T e_{1-x}$ Alloyed-Crown Region. Anisotropic growth mixture was prepared according to a slightly modified recipe. For a typical cadmium precursor preparation, $480 \mathrm{mg}$ of cadmium acetate dihydrate, $340 \mu \mathrm{L}$ of $\mathrm{OA}$, and $2 \mathrm{~mL}$ of ODE were mixed in a three-necked flask. The solution was heated to $150{ }^{\circ} \mathrm{C}$ under ambient conditions with rigorous stirring while also regularly sonicating. After the formation of a homogeneous gel with whitish color, the cadmium precursor was cooled to room temperature and stored for the crown coating. Te-TOP-ODE (0.03 M) solution was used for the tellurium ( $\mathrm{Te}$ ) precursors. It was obtained by the dilution of Te-TOP $(1 \mathrm{M})$ solution with ODE and prepared inside the glovebox. Se-TOP-ODE (0.03 M) solution was used for the selenium (Se) precursors. It was obtained by the dilution of Se-TOP $(1 \mathrm{M})$ solution with ODE and prepared inside the glovebox.

Synthesis of $4 \mathrm{ML}$ Thick Core/Alloyed-Crown CdSe/CdSe $e_{x} \mathrm{Te}_{1-x}$ $C Q W s$. For typical crown coating, $2 \mathrm{~mL}$ of $4 \mathrm{ML}$ thick CdSe CQWs dissolved in hexane (having an optical density of 0.5 at $350 \mathrm{~nm}$ ), $5 \mathrm{~mL}$ of ODE, $40 \mu \mathrm{L}$ of $\mathrm{OA}$, and $0.39 \mathrm{~mL}$ of cadmium precursors are put into a three-necked flask. First, the solution was evacuated at room temperature for the complete 
removal of hexane. Then, the solution was further evacuated around $100{ }^{\circ} \mathrm{C}$ for the removal of other residuals including water and any other organic solvents. Following that, the temperature of the solution was increased to $240{ }^{\circ} \mathrm{C}$ under an argon flow for the coating of the crown region. When the temperature reached $240{ }^{\circ} \mathrm{C}$, a desired amount of tellurium precursor (Te-TOP-ODE $(0.03 \mathrm{M})$ ) started to be injected at the rate of $8 \mathrm{~mL} / \mathrm{h}$. After the complete injection of Te precursor, the synthesis was further annealed for $5 \mathrm{~min}$ at 240 ${ }^{\circ} \mathrm{C}$. Finally, the reaction was stopped with the injection of $1 \mathrm{~mL}$ of OA and cooled to room temperature. In addition, depending on the injection amount of Te precursor (between 1.0-2.0 $\mathrm{mL}$ ), different sizes of the CdTe crown region can be grown.

For the synthesis of $\mathrm{CdSe}_{x} \mathrm{Te}_{1-x}$ alloyed-crown region, we also followed the same recipe with the synthesis of pure CdTe crown region. Simply changing the ratio of $\mathrm{Se}$ to $\mathrm{Te}$ in the injection precursor, we performed the synthesis with the same amount of injection, which is used for the growth of pure CdTe crown region. For the samples used in these studies, $1.5 \mathrm{~mL}$ of injection precursors having different ratios ( $\mathrm{Te}$ to $\mathrm{Se}$ ) was used. For the further characterization of $\mathrm{CdSe} / \mathrm{CdTe}$ core/crown and core/alloyed crown CQWs, they were purified with successive precipitation and finally dispersed in hexane.

PL QE measurement: We compared the emission intensity to Rho6G having 95\% PL QE.

\section{ASSOCIATED CONTENT}

\section{S Supporting Information}

The Supporting Information is available free of charge on the ACS Publications website at DOI: 10.1021/acs.jpclett.7b02367.

TEM of the core-only CQWs, XPS analysis of the alloyed-crown composition, photograph of the spincoated and encapsulated samples, statistical distribution of the ASE thresholds, and photoluminescence lifetime components and their contributions. (PDF)

\section{AUTHOR INFORMATION}

\section{Corresponding Authors}

*B.G.: E-mail: guzelturk@gmail.com.

*H.V.D.: E-mail: hvdemir@ntu.edu.sg; volkan@bilkent.edu. tr. Tel: +65 67905395 .

\section{ORCID}

Burak Guzelturk: 0000-0003-1977-6485

Tianquan Lian: 0000-0002-8351-3690

Hilmi Volkan Demir: 0000-0003-1793-112X

\section{Present Address}

${ }^{\perp}$ B.G.: Stanford Institute for Materials and Energy Sciences, SLAC National Accelerator Laboratory, Menlo Park, California 94025, USA.

\section{Notes}

The authors declare no competing financial interest.

\section{ACKNOWLEDGMENTS}

We acknowledge the financial support partly from National Research Foundation, Prime Minister's Office, Singapore under its NRF Investigatorship Award (NRF-NRFI2016-08) and A*STAR SERC PHAROS Program (152 73 00025) of Singapore and partly from EU-FP7 Nanophotonics4Energy NoE and TUBITAK 114F326 and 114E449. H.V.D. acknowledges additional support from ESF-EURYI and TUBA, and Y.K. acknowledges support from TUBITAK-BIDEB. T.L. also gratefully acknowledges the financial support from the U.S. National Science Foundation (CHE-1309817). We thank Prof. Alexander L. Efros for the discussions on the repulsive excitonexciton interactions.

\section{REFERENCES}

(1) Guzelturk, B.; Kelestemur, Y.; Gungor, K.; Yeltik, A.; Akgul, M. Z.; Wang, Y.; Chen, R.; Dang, C.; Sun, H.; Demir, H. V. Stable and Low-Threshold Optical Gain in CdSe/CdS Quantum Dots: An AllColloidal Frequency Up-Converted Laser. Adv. Mater. 2015, 27, 2741-2746.

(2) Dang, C.; Lee, J.; Breen, C.; Steckel, J. S.; Coe-Sullivan, S.; Nurmikko, A. Red, Green and Blue Lasing Enabled by Single-Exciton Gain in Colloidal Quantum Dot Films. Nat. Nanotechnol. 2012, 7, 335-339.

(3) Klimov, V. I.; Mikhailosvsky, A. A.; Xu, S.; Malko, A.; A, H. J.; Leatherdale, C. A.; Eisler, H. J.; Bawendi, M. G. Optical Gain and Stimulated Emission in Nanocrystal Quantum Dots. Science 2000, 290, 314-317.

(4) Guzelturk, B.; Kelestemur, Y.; Akgul, M. Z.; Sharma, V. K.; Demir, H. V. Ultralow Threshold One-Photon- and Two-PhotonPumped Optical Gain Media of Blue-Emitting Colloidal Quantum Dot Films. J. Phys. Chem. Lett. 2014, 5, 2214-2218.

(5) Wang, Y.; Ta, V. D.; Gao, Y.; He, T. C.; Chen, R.; Mutlugun, E.; Demir, H. V.; Sun, H. D. Stimulated Emission and Lasing from CdSe/ CdS/ZnS Core-Multi-Shell Quantum Dots by Simultaneous ThreePhoton Absorption. Adv. Mater. 2014, 26, 2954-2961.

(6) Xing, G.; Liao, Y.; Wu, X.; Chakrabortty, S.; Liu, X.; Yeow, E. K. L.; Chan, Y.; Sum, T. C. Ultralow-Threshold Two-Photon Pumped Amplified Spontaneous Emission and Lasing from Seeded CdSe/CdS Nanorod Heterostructures. ACS Nano 2012, 6, 10835-10844.

(7) Snee, P. T.; Chan, Y.; Nocera, D. G.; Bawendi, M. G. WhisperingGallery-Mode Lasing from a Semiconductor Nanocrystal/Microsphere Resonator Composite. Adv. Mater. 2005, 17, 1131-1136.

(8) Guzelturk, B.; Martinez, P. L. H.; Zhang, Q.; Xiong, Q.; Sun, H.; Sun, X. W.; Govorov, A. O.; Demir, H. V. Excitonics of Semiconductor Quantum Dots and Wires for Lighting and Displays. Laser Photon. Rev. 2014, 8, 73-93.

(9) Wierer, J. J.; Tsao, J. Y.; Sizov, D. S. Comparison between Blue Lasers and Light-Emitting Diodes for Future Solid-State Lighting. Laser Photon. Rev. 2013, 7, 963-993.

(10) Kelestemur, Y.; Cihan, A. F.; Guzelturk, B.; Demir, H. V. TypeTunable Amplified Spontaneous Emission from Core-Seeded CdSe/ CdS Nanorods Controlled by Exciton-Exciton Interaction. Nanoscale 2014, 6, 8509-8514.

(11) Cihan, A. F.; Kelestemur, Y.; Guzelturk, B.; Yerli, O.; Kurum, U.; Yaglioglu, H. G.; Elmali, A.; Demir, H. V. Attractive versus Repulsive Excitonic Interactions of Colloidal Quantum Dots Control Blue- to Red-Shifting (and Non-Shifting) Amplified Spontaneous Emission. J. Phys. Chem. Lett. 2013, 4, 4146-4152.

(12) Grivas, C.; Li, C.; Andreakou, P.; Wang, P.; Ding, M.; Brambilla, G.; Manna, L.; Lagoudakis, P. Single-Mode Tunable Laser Emission in the Single-Exciton Regime from Colloidal Nanocrystals. Nat. Commun. 2013, 4, 2376.

(13) Kazes, M.; Oron, D.; Shweky, I.; Banin, U. Temperature Dependence of Optical Gain in CdSe/ZnS Quantum Rods. J. Phys. Chem. C 2007, 111, 7898-7905.

(14) Dang, C.; Nurmikko, A. Beyond Quantum Dot LEDs: Optical Gain and Laser Action in Red, Green, and Blue Colors. MRS Bull. 2013, 38, 737-742.

(15) Park, Y.-S.; Bae, W. K.; Baker, T.; Lim, J.; Klimov, V. I. Effect of Auger Recombination on Lasing in Heterostructured Quantum Dots with Engineered Core/Shell Interfaces. Nano Lett. 2015, 15, 73197328.

(16) Klimov, V. I.; Ivanov, S. A.; Nanda, J.; Achermann, M.; Bezel, I.; McGuire, J. A.; Piryatinski, A. Single-Exciton Optical Gain in Semiconductor Nanocrystals. Nature 2007, 447, 441-446. 
(17) Nanda, J.; Ivanov, S. A.; Achermann, M.; Bezel, I.; Piryatinski, A.; Klimov, V. I. Light Amplification in the Single-Exciton Regime Using Exciton-Exciton Repulsion in Type-II Nanocrystal Quantum Dots. J. Phys. Chem. C 2007, 111, 15382-15390.

(18) Ithurria, S.; Dubertret, B. Quasi 2D Colloidal CdSe Platelets with Thicknesses Controlled at the Atomic Level. J. Am. Chem. Soc. 2008, 130, 16504-16505.

(19) Ithurria, S.; Tessier, M. D.; Mahler, B.; Lobo, R. P. S. M.; Dubertret, B.; Efros, A. L. Colloidal Nanoplatelets with TwoDimensional Electronic Structure. Nat. Mater. 2011, 10, 936-941.

(20) Lhuillier, E.; Pedetti, S.; Ithurria, S.; Nadal, B.; Heuclin, H.; Dubertret, B. Two-Dimensional Colloidal Metal Chalcogenides Semiconductors: Synthesis, Spectroscopy, and Applications. Acc. Chem. Res. 2015, 48, 22-30.

(21) She, C.; Fedin, I.; Dolzhnikov, D. S.; Demortière, A.; Schaller, R. D.; Pelton, M.; Talapin, D. V. Low-Threshold Stimulated Emission Using Colloidal Quantum Wells. Nano Lett. 2014, 14, 2772-2777.

(22) Guzelturk, B.; Kelestemur, Y.; Olutas, M.; Delikanli, S.; Demir, H. V. Amplified Spontaneous Emission and Lasing in Colloidal Nanoplatelets. ACS Nano 2014, 8, 6599-6605.

(23) Grim, J. Q.; Christodoulou, S.; Di Stasio, F.; Krahne, R.; Cingolani, R.; Manna, L.; Moreels, I. Continuous-Wave Biexciton Lasing at Room Temperature Using Solution-Processed Quantum Wells. Nat. Nanotechnol. 2014, 9, 891-895.

(24) Kelestemur, Y.; Dede, D.; Gungor, K.; Usanmaz, C. F.; Erdem, O.; Demir, H. V. Alloyed Heterostructures of CdSexS1-X Nanoplatelets with Highly Tunable Optical Gain Performance. Chem. Mater. 2017, 29, 4857.

(25) Pedetti, S.; Ithurria, S.; Heuclin, H.; Patriarche, G.; Dubertret, B. Type-II Core/Crown CdSe/CdTe Semiconductor Nanoplatelets. J. Am. Chem. Soc. 2014, 136, 16430-16438.

(26) Kelestemur, Y.; Olutas, M.; Delikanli, S.; Guzelturk, B.; Akgul, M. Z.; Demir, H. V. Type-II Colloidal Quantum Wells: CdSe/CdTe Core/Crown Heteronanoplatelets. J. Phys. Chem. C 2015, 119, 21772185.

(27) Wu, K.; Li, Q.; Jia, Y.; McBride, J. R.; Xie, Z.; Lian, T. Efficient and Ultrafast Formation of Long-Lived Charge-Transfer Exciton State in Atomically Thin Cadmium Selenide/Cadmium Telluride Type-II Heteronanosheets. ACS Nano 2015, 9, 961-968.

(28) Antanovich, A. V.; Prudnikau, A. V.; Melnikau, D.; Rakovich, Y. P.; Chuvilin, A.; Woggon, U.; Achtstein, A. W.; Artemyev, M. V. Colloidal Synthesis and Optical Properties of Type-II CdSe-CdTe and Inverted CdTe-CdSe Core-Wing Heteronanoplatelets. Nanoscale 2015, 7, 8084-8092.

(29) Li, Q.; Xu, Z.; McBride, J. R.; Lian, T. Low Threshold Multiexciton Optical Gain in Colloidal CdSe/CdTe Core/Crown Type-II Nanoplatelet Heterostructures. ACS Nano 2017, 11, 25452553.

(30) Pedetti, S.; Ithurria, S.; Heuclin, H.; Patriarche, G.; Dubertret, B. Type-II CdSe/CdTe Core/Crown Semiconductor Nanoplatelets. J. Am. Chem. Soc. 2014, 136, 16430-16438.

(31) Mahler, B.; Nadal, B.; Bouet, C.; Patriarche, G.; Dubertret, B. Core/shell Colloidal Semiconductor Nanoplatelets. J. Am. Chem. Soc. 2012, 134, 18591-18598.

(32) Bailey, R. E.; Nie, S. Alloyed Semiconductor Quantum Dots: Tuning the Optical Properties Without Changing the Particle Size. J. Am. Chem. Soc. 2003, 125, 7100-7106.

(33) Scholes, G. D.; Jones, M.; Kumar, S. Energetics of Photoinduced Electron-Transfer Reactions Decided by Quantum Confinement. J. Phys. Chem. C 2007, 111, 13777-13785.

(34) Boldt, K.; Schwarz, K. N.; Kirkwood, N.; Smith, T. A.; Mulvaney, P. Electronic Structure Engineering in $\mathrm{ZnSe} / \mathrm{CdS}$ Type-II Nanoparticles by Interface Alloying. J. Phys. Chem. C 2014, 118, 13276-13284.

(35) García-Santamaría, F.; Chen, Y.; Vela, J.; Schaller, R. D.; Hollingsworth, J. a; Klimov, V. I. Suppressed Auger Recombination in "Giant" Nanocrystals Boosts Optical Gain Performance. Nano Lett. 2009, 9, 3482-3488.
(36) Nanda, J.; Ivanov, S. a.; Achermann, M.; Bezel, I.; Piryatinski, a.; Klimov, V. I. Light Amplification in the Single-Exciton Regime Using Exciton-Exciton Repulsion in Type-II Nanocrystal Quantum Dots. J. Phys. Chem. C 2007, 111, 15382-15390.

(37) Benchamekh, R.; Gippius, N. a.; Even, J.; Nestoklon, M. O.; Jancu, J.-M.; Ithurria, S.; Dubertret, B.; Efros, A. L.; Voisin, P. TightBinding Calculations of Image-Charge Effects in Colloidal Nanoscale Platelets of CdSe. Phys. Rev. B: Condens. Matter Mater. Phys. 2014, 89, 35307.

(38) Naeem, A.; Masia, F.; Christodoulou, S.; Moreels, I.; Borri, P.; Langbein, W. Giant Exciton Oscillator Strength and Radiatively Limited Dephasing in Two-Dimensional Platelets. Phys. Rev. B: Condens. Matter Mater. Phys. 2015, 91, 121302.

(39) She, C.; Fedin, I.; Dolzhnikov, D. S.; Dahlberg, P. D.; Engel, G. S.; Schaller, R. D.; Talapin, D. V. Red, Yellow, Green, and Blue Amplified Spontaneous Emission and Lasing Using Colloidal CdSe Nanoplatelets. ACS Nano 2015, 9, 9475.

(40) Saba, M.; Minniberger, S.; Quochi, F.; Roither, J.; Marceddu, M.; Gocalinska, A.; Kovalenko, M. V.; Talapin, D. V.; Heiss, W.; Mura, A.; et al. Exciton-Exciton Interaction and Optical Gain in Colloidal CdSe/CdS Dot/Rod Nanocrystals. Adv. Mater. 2009, 21, 4942-4946.

(41) Shaklee, K. L.; Nahory, R. E.; Leheny, R. F. Optical Gain in Semiconductors. J. Lumin. 1973, 7, 284-309.

(42) Sargent, E. H.; Konstantatos, G. Colloidal Quantum Dot Optoelectronics and Photovoltaics; Konstantatos, G., Sargent, E. H., Eds.; Cambridge University Press: Cambridge, U.K., 2013; Vol. 7.

(43) Koechner, W. Solid-State Laser Engineering; Springer Series in Optical Sciences; Springer: New York, 2006; Vol. 1.

(44) Li, M.; Zhi, M.; Zhu, H.; Wu, W.-Y.; Xu, Q.-H.; Jhon, M. H.; Chan, Y. Ultralow-Threshold Multiphoton-Pumped Lasing from Colloidal Nanoplatelets in Solution. Nat. Commun. 2015, 6, 8513.

(45) Guzelturk, B.; Erdem, O.; Olutas, M.; Kelestemur, Y.; Demir, H. V. Stacking in Colloidal Nanoplatelets: Tuning Excitonic Properties. ACS Nano 2014, 8, 12524-12533. 